\title{
Opinion dynamics of random-walking agents on a lattice
}

\author{
Suhan Ree* \\ Department of Industrial Information, Kongju National University, Yesan-Up, Yesan-Gun, Chungnam, 340-702, South Korea and \\ Center for Complex Quantum Systems and Department of Physics, University of Texas at Austin, Austin, Texas 78712, USA
}

(Received 6 January 2011; published 16 May 2011)

\begin{abstract}
The opinion dynamics of random-walking agents on finite two-dimensional lattices is studied. In the model, the opinion is continuous, and both the lattice and the opinion can be either periodic or nonperiodic. At each time step, all agents move randomly on the lattice, and update their opinions based on those of neighbors with whom the differences of opinion are not greater than a given threshold. Due to the effect of repeated averaging, opinions first converge locally, and eventually reach steady states. As in other models with bounded confidence, steady states in general are those with one or more opinion groups in which all agents have the same opinion. When both the lattice and the opinion are periodic, however, metastable states can emerge, in which the whole spectrum of location-dependent opinions can coexist. This result shows that, when a set of continuous opinions forms a structure like a circle, unlike the typically used linear opinions, rich dynamic behavior can arise. When there are geographical restrictions in real situations, a complete consensus is rarely reached, and metastable states here might be one of the explanations for these situations, especially when opinions are not linear.
\end{abstract}

DOI: 10.1103/PhysRevE.83.056110

PACS number(s): 89.75.Fb, 87.23.Ge, 02.50.Ey, 05.40.Fb

\section{INTRODUCTION}

The attempt to investigate social systems by physicists is several decades old [1], even though social dynamics has become a popular subject in statistical physics only recently [2]. One of the reasons for this interest is that one basic approach to study social systems is similar to what statistical physicists typically try to do: namely, finding macroscopic behavior or emergence from the dynamics of microscopic entities $[3,4]$. While physical systems deal with particles, entities that make up social systems are humans, or groups of humans. Figuring out the dynamic behavior of even one human being is not an easy task, but some aspects of collective behavior of many individuals are known to be describable using microscopic models [3], and might even be universal [5]. In addition, due to the current ubiquity of the internet, especially the popularity of social networks, and the increased capability of processing vast amounts of social data, this kind of approach has become not only possible but also useful.

Opinion dynamics is one of the social-dynamics problems that can be closely related to physical problems. The microscopic models we are interested in here typically evolve with discrete time steps, and have a fixed number of "agents" (actors or individuals) with their own opinions. We can categorize these models using several basic features. Opinions can be discrete [6-16] or continuous [12,17-25]. Examples of discrete opinions are "yes" or "no" on a question (two values), evaluation on a scale from 1 to 5 (five values), choices in elections (two or more values), and so on. When there are more than a few choices, however, continuous opinions can be used: fine-scaled evaluation of something on a scale from 0 to 1 , political views, and so on. An opinion can be a vector of integers [19,26-28] as well. Another important feature is how a model restricts interacting partners of an agent at a given time. Agents typically have ongoing relationships with others, and interact with peers selected from

*suhan@physics.utexas.edu the related agents. The structure of these relationships plays an important role in social dynamics, and networks can be used to describe these relations (social networks). We can divide models into three different cases: (i) fully connected networks, where each agent is related to all other agents at any moment (there is no restriction, and the network concept is not necessary) [12,18-20,27-29]; (ii) fixed networks, where each agent is related to the limited number of agents given by time-independent networks [6,9-11,19-21,26,27]; (iii) evolving networks, where network structures evolve with time [13-17,22,24,25,30]. Finally, models can be differentiated by how the updating agents are chosen at each time step. One can update one agent at a time (the serial update), or all agents synchronously, especially when the order of update does not play a role (the parallel update) [18,21].

The model introduced here uses continuous opinions with evolving networks, and the parallel update. Agents reside and move randomly on a two-dimensional (2D) lattice. At each time step, agents update their locations in the lattice using the 2D random walk, and change their opinions synchronously. Only nearest-neighbor interactions are allowed for opinion changes; hence interacting partners can be represented by a contact network [31], whose evolution is only governed by the movements of agents. This is basically the process of repeated averaging [17,32], and opinions have a tendency to move toward those of the neighbors. Our model also uses a threshold to restrict interactions between agents with a big difference of opinions (bounded confidence; its use can be justified in many aspects $[33,34])$. In most models with the above setup [11,12,18-21,23,24,29,35], the system eventually reaches a steady state, where one or more groups of agents reach their consensus. Unlike other models, we assume that both the lattice and the opinion can be periodic. The shape of the lattice can be either rectangular or toroidal: two of the simplest shapes, and yet different topologically. Opinions can be periodic, too, when an opinion is about a periodic subject like the time of the year. When both the lattice and the opinions are periodic, we observe some periodic metastable states. 
In these states, there is no consensus even though opinions converge locally, and the whole spectrum of opinions, which depend only on spatial locations of agents, can coexist.

The plan of this paper is as follows. In Sec. II, the model is described, and steady states of an extreme case of a onedimensional lattice are found. In Sec. III, numerical results when both the lattice and the opinion are periodic are shown. Finally, in Sec. IV, possible extensions and many aspects of this model are discussed.

\section{MODEL}

We propose a simple model for the opinion dynamics of random-walking agents with only nearest-neighbor interactions. We assume that there are $N$ agents, each with an opinion, and that they reside on finite $2 \mathrm{D}$ lattices. Opinion changes will come only from interactions with neighbors. Time is discrete and is represented by a dimensionless quantity $t$, which is a non-negative integer. Agent $i(1 \leqslant i \leqslant N)$ will have a location and an opinion at time $t$. Because the structure of the lattice can play an important role in the dynamics, we consider two structures: a rectangle (nonperiodic) and a 2D torus (periodic) [see Fig. 1(a)]. The location on the lattice for agent $i$ at time $t$ is $\left(x_{i}, y_{i}\right)$, where $x_{i}$ and $y_{i}$ are non-negative integers $\left(0 \leqslant x_{i}<X\right.$ and $0 \leqslant y_{i}<Y$ ).

In our model, an opinion of agent $i, \phi_{i}$, is a real number between 0 and $1\left(\phi_{i} \in[0,1]\right)$, and can be either periodic or nonperiodic [see Fig. 1(b)]. For nonperiodic opinions, $\phi_{i}$ is a number on a line between 0 and 1 ; while, for periodic opinions, $\phi_{i}$ is a point on a circle (for this case, 0 and 1 are the same opinion). Then, the state of agent $i$ at time $t, s_{i}(t)$, is represented by three numbers,

$$
s_{i}(t)=\left[x_{i}(t), y_{i}(t) ; \phi_{i}(t)\right],
$$

and the state of $N$ agents is an $N$-tuple of $s_{i}$ 's,

$$
s=\left(s_{1}, s_{2}, \ldots, s_{N}\right) .
$$

How do states of agents evolve? The change of $s_{i}$ will depend on states of other agents. For each agent, there are
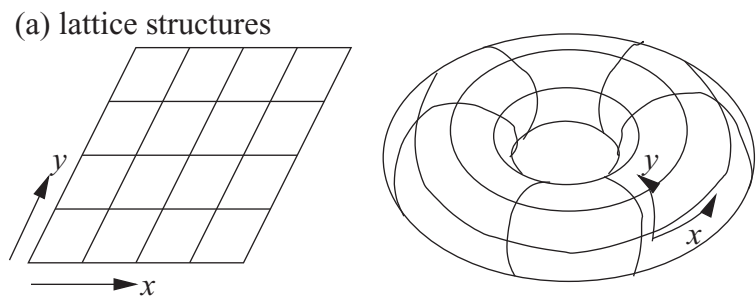

(b) opinion structures
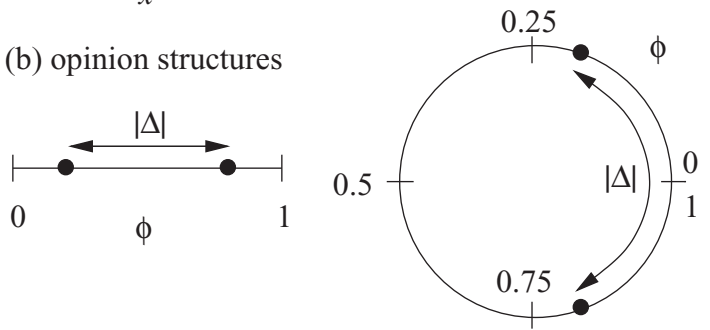

FIG. 1. Structures of the lattice and the opinion. Note that, for example, the differences of opinions between 0.2 and 0.8 are different for the two cases: 0.6 and 0.4 , respectively. two kinds of movement: changing locations in the lattice and change of opinions. First, we look at how agents move in lattices. For simplicity, we use an independent random-walk motion for each agent. The movement of agent $i(1 \leqslant i \leqslant N)$ is represented by possible choices of locations at the next time step as follows:

$$
\left(x_{i}, y_{i}\right) \rightarrow\left\{\begin{array}{l}
\left(x_{i}+1, y_{i}\right), \\
\left(x_{i}, y_{i}+1\right), \\
\left(x_{i}-1, y_{i}\right), \\
\left(x_{i}, y_{i}-1\right), \\
\left(x_{i}, y_{i}\right) .
\end{array}\right.
$$

Here an agent can move to five neighboring locations, including an option for staying ${ }^{1}$ with equal probabilities, $1 / 5$. If an agent has fewer than five choices for movement, it will move to one of the possible locations with equal probabilities as well. For the rectangular lattice, for example, if an agent is on an edge, it has four possible choices with equal probabilities, $1 / 4$. We assume that more than one agent can reside in the same location, and we define neighbors as agents residing at the same location at a given time. Since agents move randomly, neighbors of an agent will also change with time. This contact network consists of disconnected cliques of various sizes as in Ref. [31].

Interacting partners of an agent are further reduced by the use of the threshold $d$ as in other models of bounded confidence $[11,12,18-21,23,24,29,35]$. If the difference of opinions for a given pair of agents is greater than $d$ there will be no influence. In other words, if we introduce $\Delta_{j i}$ as the difference of opinions between agents $i$ and $j$, agent $i$ will not be influenced by agent $j$ if $\left|\Delta_{j i}\right|$ is greater than $d$. For nonperiodic opinions, $\Delta_{j i}$ can be obtained by subtraction,

$$
\Delta_{j i}=\phi_{j}-\phi_{i} .
$$

For periodic opinions, on the other hand, we set

$$
\Delta_{j i}= \begin{cases}\phi_{j}-\phi_{i}+1 & \left(\text { if } \phi_{j}-\phi_{i} \leqslant-0.5\right), \\ \phi_{j}-\phi_{i}-1 & \left(\text { if } \phi_{j}-\phi_{i}>0.5\right), \\ \phi_{j}-\phi_{i} & \text { (otherwise) }\end{cases}
$$

[see Fig. 1(b)]. The ranges of both $\Delta_{j i}$ and $d$ are different for two types of opinion: for nonperiodic opinions, $-1 \leqslant \Delta_{j i} \leqslant 1$ and $0<d \leqslant 1$, and for periodic opinions, $-0.5<\Delta_{j i} \leqslant 0.5$ and $0<d<0.5$ ( $d=0.5$ for periodic opinions is excluded because it can introduce an uncertainty).

Then, we can define the set of neighbors with similar opinions as $K_{i}$, where agents in this set are neighbors of agent $i$ at a given time and the absolute values of the differences of their opinions with agent $i$ are less than or equal to $d$. By including agent $i$ in $K_{i}\left(i \in K_{i}\right)$, there will be at least one element in $K_{i}$. Then, the opinion of agent $i, \phi_{i}$, at $t+1$ becomes

$$
\phi_{i}(t+1)=\phi_{i}(t)+g \frac{\sum_{j \in K_{i}(t)} \Delta_{j i}(t)}{\left|K_{i}(t)\right|},
$$

\footnotetext{
${ }^{1}$ If $X$ and $Y$ are even numbers, some agents will never meet at the same location if only four moving choices are given in Eq. (3).
} 
where $g$ is a convergence parameter $(0<g \leqslant 1)$, and $\left|K_{i}(t)\right|$ is the number of elements in the set $K_{i}$ at time $t$. When $\left|K_{i}\right|=1$, there is no interaction for agent $i$, and $\phi_{i}$ will not change. When $\left|K_{i}\right|=2$, the interaction is binary, and Eq. (6) becomes the equation in the Deffuant model [29] (in the original model, $\mu$ was used, which is basically $g / 2$ ). When $\left|K_{i}\right|>2$, agent $i$ is interacting with more than one neighbor at once as in the Hegselmann-Krause model [18].

The right-hand side of Eq. (6) can also be written as $\phi_{i}+g\left(\bar{\phi}_{i}-\phi_{i}\right)$, where $\bar{\phi}_{i}$ is the average opinion of agents in $K_{i}$. When $g=1$ and $d$ is maximal, all interacting agents will have the same averaged opinion at the next step. For example, for nonperiodic opinions, when two agents with $\phi_{1}$ and $\phi_{2}$ interact, both of their opinions will become $\left(\phi_{1}+\phi_{2}\right) / 2$ at the next step. Note that care has to be taken when the opinion is periodic. For example, the average of opinions 0.1 and 0.9 should be 0 , not 0.5 as in the nonperiodic case. For periodic opinions, the value of $\phi_{i}$ can become greater than 1 or less than 0 after Eq. (6) is applied; in those cases, we can adjust the $\phi_{i}$ value by subtracting or adding 1 to keep $\phi_{i}$ between 0 and 1 . When $0<g<1$, every interacting agent will move toward a certain value at the next step. If the same agents interact for more than one time step, their opinions will gradually converge to one opinion value. The bigger the value of $g$, the faster they will converge. We are not considering the case with $g=0$ or $d=0$, because the model becomes trivial. This model has five parameters: $N, X, Y, d$, and $g$.

At $t=0$, the state of all agents is given, and opinions and locations thereafter will be calculated from the state of the previous time step. At each time step, agents undergo random walks on the lattice and change opinions according to Eq. (6) synchronously. This dynamic process is stochastic because of random walks, even though opinion changes are deterministic. This also is a Markov process because the state at the previous time step is all we need to find the current state and beyond.

Before looking at numerical results, we can get some insights by looking at an extreme case, the one-dimensional (1D) case (by setting $Y$ as 1 ) with $g=1$ and the maximal $d$. In this case, the opinions of agents at one location converge to the same value at each time step according to Eq. (6), and due to local interactions, the whole state can be approximated by a continuous $1 \mathrm{D}$ curve in $(x, \phi)$ space [see Fig. 2(a)]. Then we can focus only on the dynamics of this curve instead of $s$ in Eq. (2). Local stability at a location can be achieved if the curve is locally linear (see the Appendix for details), and we get global stability when the curve is linear everywhere, which means that steady states are straight lines in $(x, \phi)$. Given an initial condition, the system will eventually reach one of the states represented by straight lines (it can be seen as a straightening process of a curve), and opinion values with respect to $x$ will not change. The most common is the one where every agent has the same opinion (complete consensus), as most models of opinion dynamics have found. Once it is reached, the opinions of all agents will not change afterward. We will call these steady states flat here, because they can be represented by flat lines in $(x, \phi)$. If we use the term an "opinion group" (a cluster or a party) for a set of agents that have reached a consensus, there will be only one opinion group for maximal $d$, while there can be more than one opinion group

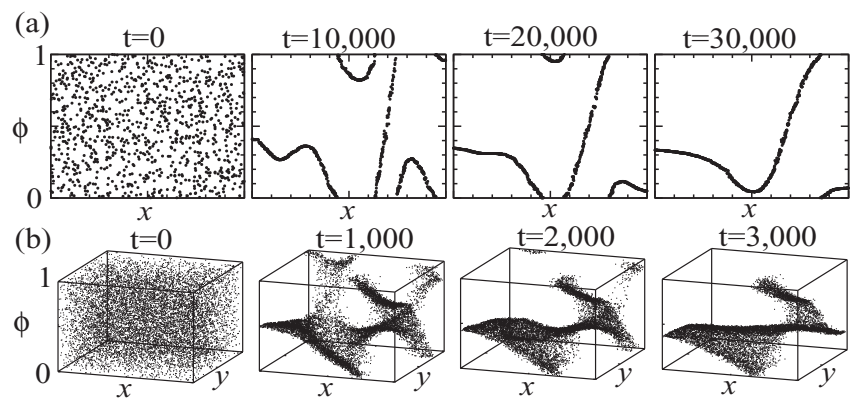

FIG. 2. Scatter plots to show time evolutions in $1 \mathrm{D}$ and $2 \mathrm{D}$ cases with nonperiodic lattice and periodic opinions. (a) 1D case: $N=$ 1000, $X=1000, Y=1, g=1 . d=0.49$. (b) $2 \mathrm{D}$ case: $N=10000$, $X=100, Y=100, g=1$, and $d=0.49$.

when $d$ is small (say, $d<0.3$ ), as seen in models of bounded confidence.

When both the lattice and the opinion are periodic, however, nonflat steady states can appear. They are called nonflat because they can be represented in $(x, \phi)$ as lines with nonzero slopes. Unlike the lifetimes of flat steady states, those of these states are finite in general depending on some parameters (which will be discussed in detail later); therefore, these states will also be called metastable states (see Ref. [16] for another type of metastable state). Note that nonflat states cannot be sustained in nonperiodic cases due to the boundary effect. ${ }^{2}$

It is not hard to generalize the $1 \mathrm{D}$ results to $2 \mathrm{D}$ lattices, and steady states will be represented by $2 \mathrm{D}$ planes in $(x, y, \phi)$ space. As in $1 \mathrm{D}$ cases, transient states will look like curved $2 \mathrm{D}$ surfaces mostly, but eventually the system will reach one of the steady states, however long it takes [see Fig. 2(b)]. When both the lattice and the opinion are periodic, nonflat metastable states can emerge, while there will be only flat steady states otherwise. When $g<1$, opinions at a location can have more than one value: in other words, at a given location there will be a distribution of opinions. For nonflat steady states, the width of the opinion distributions at a given location will be finite, and as $g$ gets smaller, the width of this distribution will increase.

\section{NUMERICAL RESULTS FOR THE PERIODIC CASE}

The toroidal (periodic) lattice with periodic opinions has richer dynamic behavior than other cases, as we saw in the previous section. In addition to the flat steady states, this system can have nonflat metastable states due to the periodicity of both the location and the opinion. We can categorize these nonflat states with period numbers with "period $n_{\phi} / n_{l}$ " ( $n_{\phi}, n_{l}=1,2,3, \ldots$; see the Appendix for details). For 2D cases, only one direction (either $x$ or $y$ ) can be periodic. Even though any period- $\left(n_{\phi} / n_{l}\right)$ steady states can exist, we observed mostly steady states of period $n_{\phi}\left(n_{l}=1\right)$ and period $1 / n_{l}$

\footnotetext{
${ }^{2}$ If opinions at the boundary and those at the location right next to the boundary are not the same, opinions at the boundary will have a tendency to move toward the opinions at the neighboring site. Therefore, for the whole state to be stable, the state has to be flat everywhere.
} 


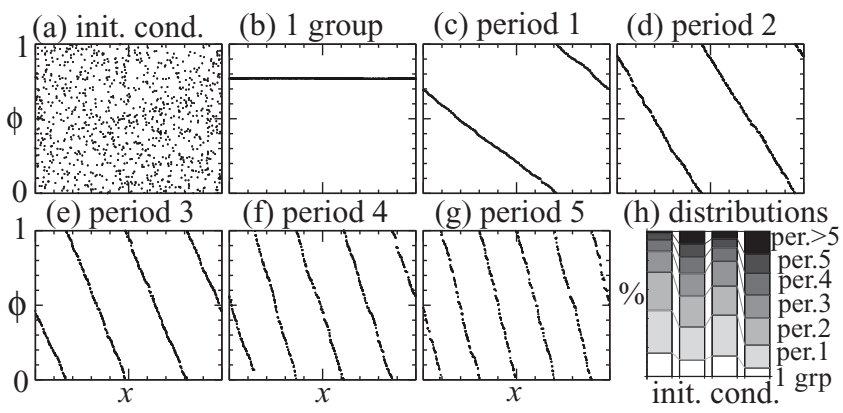

FIG. 3. Scatter plots for the case with $1 \mathrm{D}$ periodic lattice and periodic opinions $(N=1000, X=1000, Y=1, g=1$, and $d=$ 0.49). (a) A randomly chosen initial condition; (b)-(g) observed steady states using the same initial condition as in (a); (h) a distribution of steady states using four randomly chosen initial conditions (using 1000 runs each).

$\left(n_{\phi}=1\right)$ in our numerical simulations, and we will show them in later figures. For all numerical results except the one in Fig. 7(a), we assume that the density of agents is always one per location.

In Fig. 3, we observe steady states that can emerge for $N=1000, X=1000, Y=1, g=1$, and $d=0.49$, with one randomly chosen initial condition $(d=0.49$ is used as a maximal value instead of 0.5 because 0.5 is excluded as was discussed in the previous section). Since the dynamics is stochastic, the system can reach different types of steady states with the same initial condition. In addition to the flat steady states [Fig. 3(b)], nonflat steady states also appear [Figs. 3(c)-3(g)]. By repeating simulations using different sets of random numbers, we can find the distribution of types of steady states when we start from one given initial condition. The distributions from different initial conditions do not have to be the same, as we show in Fig. 3(h) for four different randomly chosen initial conditions.

How do we find the dynamical properties of a system with given parameters? Each initial condition will have its own distribution as we saw in Fig. 3; therefore the distribution we obtain after averaging over those from all initial conditions characterizes the system. The more samples we choose and the more runs we perform for each sample, the more accurate this distribution should be. For each parameter set used subsequently, we will sample 1000 initial conditions randomly from the space of all initial conditions (in this case, $\left.\left\{\left(x_{i}, y_{i}, \phi_{i}\right) \mid 1 \leqslant i \leqslant N\right\}\right)$, and run once each, to find the approximate distribution (the total of 1000 runs).

For $2 \mathrm{D}$ cases, the results are similar to those from 1D cases. In Fig. 4, we looked at steady states for two different cases: $(X, Y)=(100,100)$ and $(100,10)$. In the first case, we observed two types of steady state: flat $(\sim 99 \%)$ and period 1 ( $\sim 1 \%)$; while, in the second case, we observed four types of steady state: flat $(\sim 38 \%)$, period $1(\sim 48 \%)$, period $2(\sim 12 \%)$, and period $3(\sim 2 \%)$. As we will show later in Fig. 7(c), the width of the lattice in the $y$ direction, $Y$, can change the dynamical behavior of the system, when $X$ is fixed. When $X=Y$, complete consensus was reached in almost all cases.

Let us next observe how the threshold $d$ changes the dynamic behavior of the system. In Fig. 5(a), we compare our results with those from the Deffuant model by observing (a) $100 \times 100$

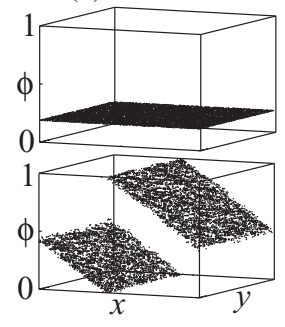

(b) $100 \times 10$

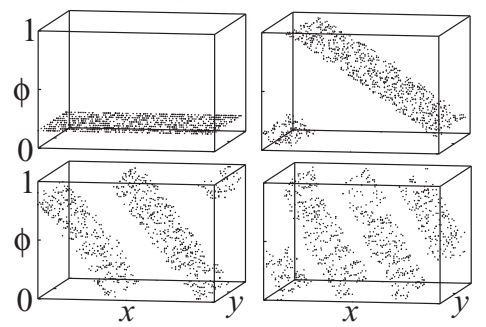

FIG. 4. Scatter plots in two cases with $2 \mathrm{D}$ periodic lattices and periodic opinions. (a) $N=10000, X=100, Y=100, g=$ 1 , and $d=0.49$. (b) $N=1000, X=100, Y=10, g=1$, and $d=0.49$.

averaged numbers of opinion groups while varying $d$. Two agents are randomly picked at each time step, and $g$ is set to 1 . In numerical results with $N=1000$ ( $\square$ ), averaged over 1000 runs, gradual transitions were observed as the number of groups increases. We simulated for periodic opinions (o), too, and got almost the same results. Two cases from our model were also simulated: a rectangular lattice with nonperiodic opinions $(\diamond)$, and a toroidal lattice with periodic opinions $(\triangle)$ when $N=1000, X=100, Y=10, g=1$, and $d=0.49$ for 1000 runs each. The results are similar to those from the Deffuant model, but numbers of groups tend to be a little smaller.

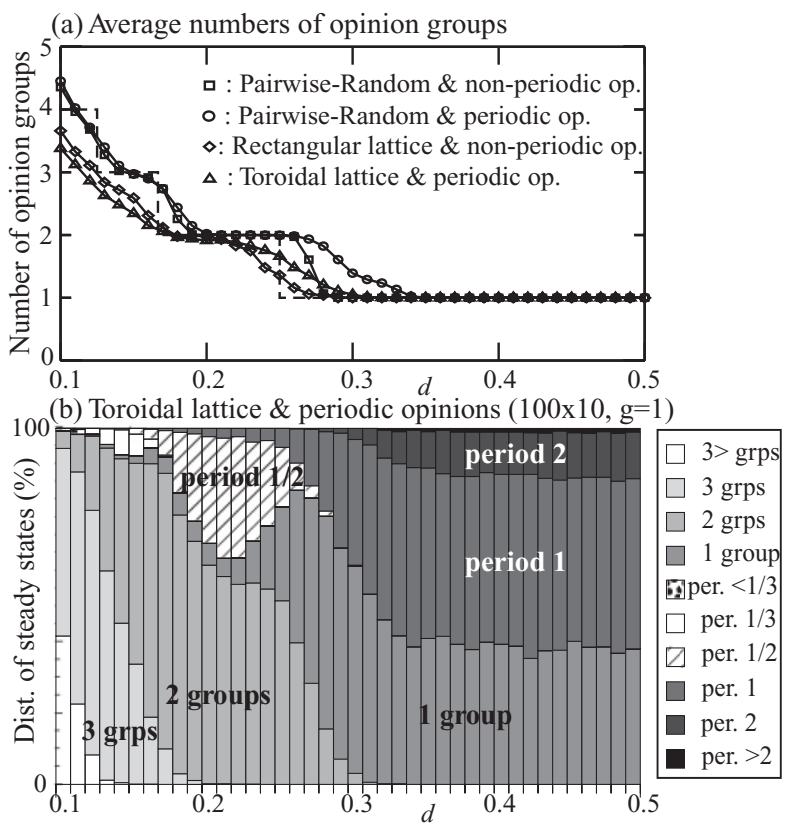

FIG. 5. (a) Number of opinion groups formed when $d$ is varied for four different settings: $N=1000, X=100, Y=10, g=1$, and $d=0.49,1000$ runs each. When counting numbers of groups, we counted groups with greater than $10 \%$ of the whole population, and ignored nonflat steady states, if they exist. The dashed line represents the prediction that, when $d$ is in the range of $1 /(n+1)<d<1 / n$, there can be $n$ opinion groups. (b) Distributions of observed steady states for the case with the toroidal lattice and periodic opinions, while $d$ is varied $(N=1000, X=100, Y=10, g=1$, and $d=0.49,1000$ runs each). 
In Fig. 5(b), steady states for the case of the toroidal lattice with periodic opinions with $N=1000, X=100, Y=10$, and $g=1$, are observed when $d$ is varied. There can be nonflat steady states. For flat steady states, we can categorize them with the number of opinion groups: one, two, three groups, and so on. When $d$ is less than $\sim 0.17$, steady states are mostly flat, and as $d$ gets smaller, more opinion groups can exist as seen in Fig. 5(a). When $d$ is between $\sim 0.17$ and $\sim 0.27$, fractional periodic states, mostly $1 / 2$, emerge, while the dominant steady states are those with two groups. When $d$ is between $\sim 0.27$ and $\sim 0.32$, this is where the transition occurs: the number of two-group steady states decreases quickly, while the number of period-1 steady states increases. When $d$ is greater than $\sim 0.32$, all flat steady states belong to the one-group type, while there can be many types of nonflat steady state. Distributions do not change much as $d$ increases up to 0.5 . In the current case, steady states with period 3 or higher do not appear much; however, when $Y / X$ is smaller, more types of periodic steady state will appear.

In Fig. 6, we show steady states in Fig. 5(b) for three $d$ values: $0.15,0.25$, and 0.35 . When $d$ is 0.15 , three types of flat steady state dominate ( $\sim 92 \%)$, while there exist nonflat steady states with fractional periods (mostly $1 / 2$ and $1 / 3$ ). When $d$ is 0.25 , more than $3 / 4$ of steady states are flat $(\sim 78 \%)$ still, while nonflat steady states with period $1 / 2$ and 1 also exist. Here there are two kinds of period-1 steady state: one has one band $\left(n_{\phi}=1\right.$ and $\left.n_{l}=1\right)$, and the other has two bands $\left(n_{\phi}=2\right.$ and $n_{l}=2$ ), which is possible because $d$ is small. When there are two bands, there are two disjoint groups of agents, even though each group has agents with a full spectrum of opinions, and they were observed only in the approximate range of $d$ between 0.18 and 0.25 . When $d$ is 0.35 , only one-group flat states were observed, and nonflat steady states of periods 1, 2, and 3 were also observed.

Finally, we can ask how other parameters will influence the outcome. In Fig. 7(a), $N$ is varied from 100 to 2000 when $X=100, Y=10, g=1$, and $d=0.49$ to observe how

(a) $d=0.15(100 \times 10, \mathrm{~g}=1)$

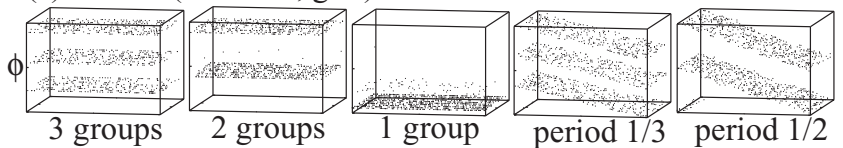

(b) $d=0.25(100 \times 10, \mathrm{~g}=1)$

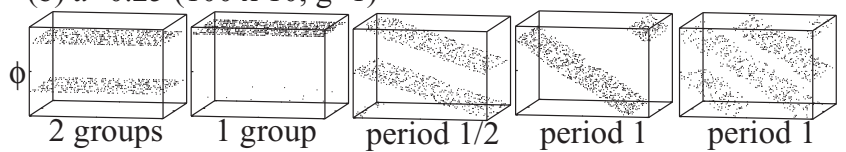

(c) $d=0.35(100 \times 10, \mathrm{~g}=1)$

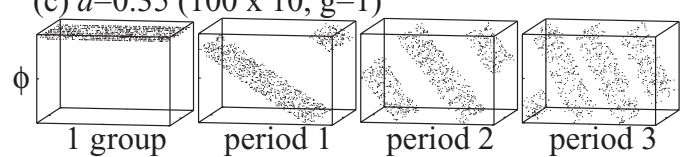

FIG. 6. Scatter plots of some observed steady states for different $d$ values from Fig. 5(b) $(N=1000, X=100, Y=10, g=1$, and $d=0.49$, toroidal lattice with periodic opinions). (a) $d=0.15$ (three groups, $34 \%$; two groups, $56 \%$; one group, $2 \%$; period $1 / 3,6 \%$; period $1 / 2,2 \%$ ); (b) $d=0.25$ (two groups, $52 \%$; one group, $26 \%$; period $1 / 2,17 \%$; period $1,5 \%$; period $1,<1 \%$ ); (c) $d=0.35$ (one group, $41 \%$; period $1,48 \%$; period $2,10 \%$; period $3,1 \%$ ).

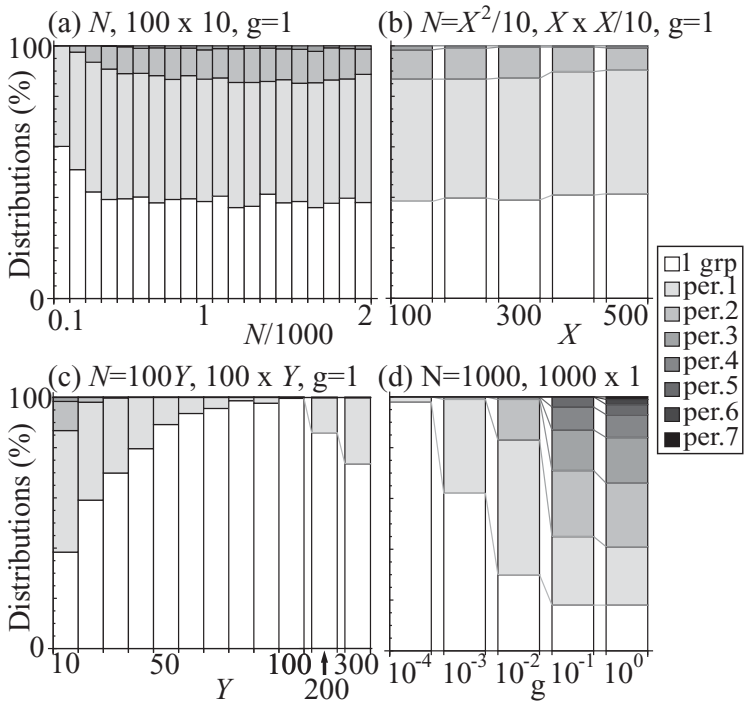

FIG. 7. Distributions of steady stateswhen $N, X, Y$, and $g$ are varied ( $d$ is fixed at 0.49 , using 1000 runs for each case). (a) Varying $N(X=100, Y=10, g=1)$. The density changes. (b) Varying $N$, $X$, and $Y(N=X Y, Y=X / 10, g=1)$. The density and $Y / X$ do not change, and the size effect can be seen. (c) Varying $N$ and $Y$ ( $N=X Y, X=100, g=1)$. The density and $X$ do not change, while the shape of the lattice changes. (d) Varying $g(N=1000, X=1000$, $Y=1)$.

the density $[N /(X Y)]$ changes dynamical behavior. When the density is much smaller than 1 , the number of nonflat steady states decreases. But we can clearly observe that if the density is greater than about 0.5 , distributions do not change much. This result shows that the density does not have to be high to find the distributions of steady states, and that the density of 1 is good enough.

In Fig. 7(b), we vary the size of the lattice while keeping the density and the shape fixed. As $X$ increases from 100 to 500 when $N=X Y, Y=X / 10, g=1$, and $d=0.49$, the distributions do not change much. We can interpret this result as a sign that there is no size effect unless the size is too small.

In Fig. 7(c), $Y$ varies from 10 to 300, changing the shape of the lattice, when $N=X Y, X=100, g=1$, and $d=0.49$. As $Y$ approaches the value of $X$, the number of nonflat steady states decreases. As we saw in Figs. 4 and 6, the periodic behavior only appears in one direction in the case of toroidal shapes. If opinions are periodic in $x$, opinions along the $y$ direction for a given $x$ value are more or less constant. When $Y$ is 10 , periodic behavior can be seen only in $x$, but as $Y$ increases, nonflat steady states along the $y$ direction start to emerge. When $X=Y$, steady states can be periodic either in $x$ or in $y$ with equal probabilities. When $Y$ is greater than $X$, the likelihood of forming steady states that are periodic in $y$ will be greater, and the probability of getting the periodic steady states starts to increase again. The more elongated the shape of the lattice, the greater is the likelihood of finding periodic steady states.

In Fig. 7(d), the convergence parameter $g$ varies when $N=$ 1000, $X=1000, Y=1$, and $d=0.49$. As $g$ gets smaller, the number of nonflat steady states decreases. To make those periodic steady states disappear, $g$ has to be very small, $\sim 10^{-4}$ 


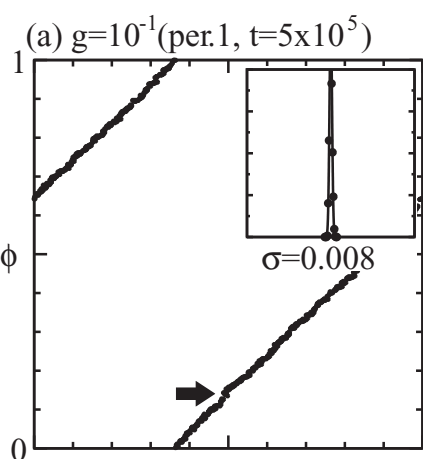

(e) $g=10^{-3}$ (per. $\left.1, \mathrm{t}=10^{6}\right)$

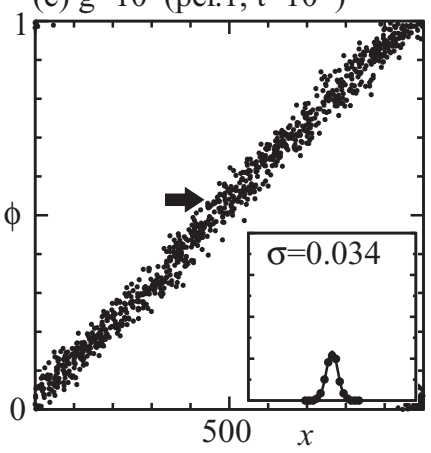

(b) $\mathrm{g}=10^{-2}$ (per. $\left.1, \mathrm{t}=5 \times 10^{5}\right)$

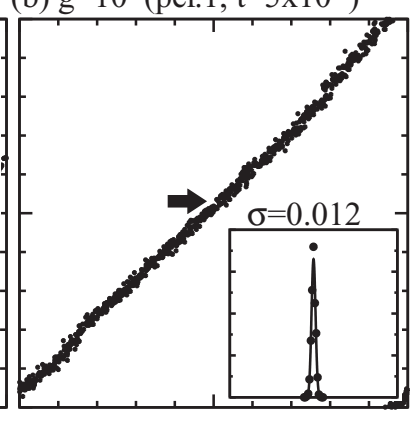

(f) $g=10^{-3}$ (per. $2, t=10^{6}$ )

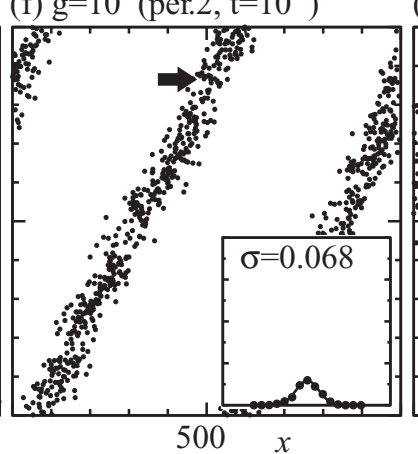

(c) $g=10^{-2}$ (per. $\left.2, \mathrm{t}=5 \times 10^{5}\right)$

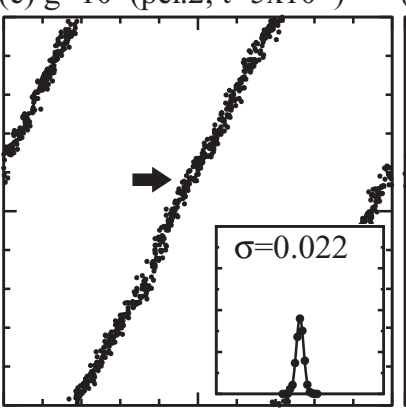

(g) $g=10^{-4}$ (per. $1, t=10^{6}$ ) (d) $g=10^{-2}\left(\right.$ per. $\left.3, t=5 \times 10^{5}\right)$

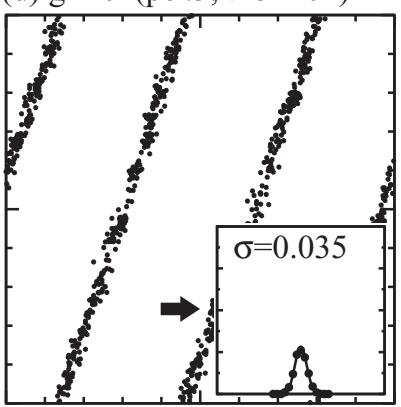

(h) $g=10^{-4}\left(3\right.$ groups, $\left.t=10^{6}\right)$

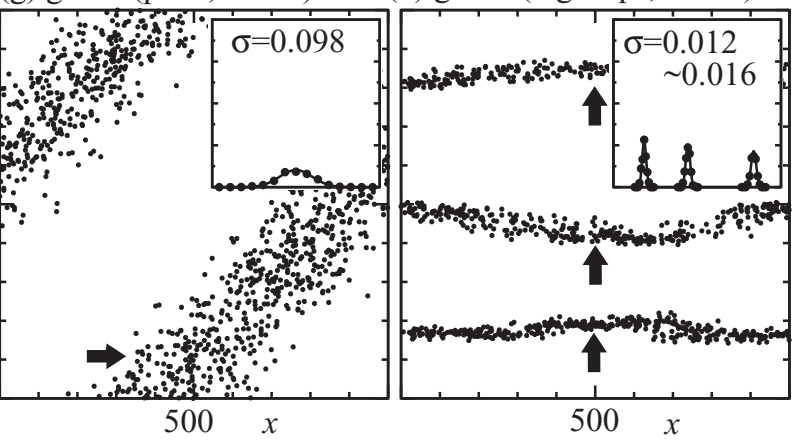

FIG. 8. Scatter plots of nonflat steady states from Fig. 7(d). The widths were obtained by fitting the distributions of $\phi$ at $x=500$ (pointed by arrows) by Gaussian functions, and $\sigma$ is the standard deviation (shown in the insets).

in this case. The convergence parameter controls how fast opinions converge; in addition, when $g$ is small, the distribution of opinions at the same location for nonflat steady states gets wider because agents can move farther away from a location without changing their opinions much.

In Fig. 8, we observe the widths of distributions of opinions at a given $x$ for some of the nonflat steady states from Fig. 7(d). In Figs. 8(a)-8(g), nonflat steady states when $g=10^{-1}, 10^{-2}$, $10^{-3}$, and $10^{-4}$ are shown. The insets show the distributions of opinions $\phi$ at $x=500$. We use the opinions of agents residing at $x=500$ at a number of different time steps after steady states are reached (for example, 1000 time steps that are 100 time steps apart). ${ }^{3}$ If these frequencies are fitted to Gaussian functions as shown in the insets in Fig. 8, the standard deviations $\sigma$ will represent the widths of these distributions. We can argue that $\sigma$ is a measure of stability. As discussed in Sec. II, we can call nonflat steady states metastable, because the lifetimes of structures formed in $(x, y, \phi)$ space are finite, but extremely long. In other words, the probability of decay within a certain finite time period is extremely small, even though it will not be zero exactly as in flat steady states. If we define $R$ as the ratio of the width of a band to the distance between two adjacent bands along the $x$ direction,

$$
R=\frac{\left(n_{l} X / n_{\phi}\right) \sigma}{X / n_{\phi}}=n_{l} \sigma
$$

\footnotetext{
${ }^{3}$ The width found in this way can be a little bigger than the actual width, because the band structures in $(x, \phi)$ graphs tend to drift a little in the $x$ direction even after the steady state has been reached. This effect has been ignored here.
}

where $n_{l} X / n_{\phi}$ is the inverse of the slope of the line in $(x, \phi)$. Since $n_{l}=1$ always in the current examples, $\sigma=R$. If $R \ll$ 0.5 , the structure is quite stable. If $R>\sim 0.5$, adjacent bands will overlap and will be broken immediately. If $R$ comes close to 0.5 , the state is likely to become flat or morph into another type of steady state in a short time period. In general, the bigger $\sigma$ is, the more unstable the state is and the less likely it will be reached spontaneously from random initial conditions.

How is $\sigma$ affected by the parameters? We have observed in Fig. 8 that the greater $g$, the smaller is $\sigma$. This width also depends on the period number $n_{\phi} / n_{l}$; for example, the width of opinions of period- 2 steady states is twice that of period-1 steady states, because the opinion difference between two adjacent locations is twice as big for period-2 steady states. Then we can generalize that for the same $g$ value, the width of opinions at a location for steady states with period $n_{\phi} / n_{l}$ is proportional to $n_{\phi} / n_{l}$. This explains why steady states with higher periods disappear quickly as $g$ gets smaller. When $g=1, \sigma$ comes close to zero unless $N$ is very small. That leads us to claim that $N$ can play a role, too: as $N$ gets smaller, $\sigma$ becomes wider because, when $N$ is small compared to $X Y$, the possibility of having empty locations increases and an agent can move further away without changing its opinion [see Fig. 7(a)]. In addition, the stability of nonflat states also depends on $Y / X$ [see Fig. 7(c)]. We can summarize based on our results so far that, in general, the stability of a certain periodic state depends directly on $N /(X Y), Y / X, g, n_{\phi}$, and $n_{l}$, while $d$ determines what types of periodic state are allowed to exist.

Figure 8(h) shows a special case. It has three opinion groups, but unlike opinion groups found in flat states when $d$ is small, they have nonzero widths of opinions and the opinion of each agent is not stationary. In addition, the lifetimes of these 
states are not long. In most cases, this type of state stayed intact for more than ten times the time it took for most steady states to be formed. This type of state was observed only in the case with $g=10^{-4}$ for about $1 \%$ of results [note that since they eventually converge to flat states, they were counted as flat states in Fig. 2(d)]. They could emerge because periodic opinions are used and opinions from three groups can be balanced in a circle. Also, $g$ has to be small enough; if $g$ is bigger, the widths of opinions will be bigger and the structure becomes unstable.

\section{DISCUSSION}

We introduced a simple model of opinion dynamics that uses only nearest-neighbor interactions. To represent the geometrical space we live in, we used 2D lattices, on which agents move randomly. Both the lattice and the opinion can be periodic or nonperiodic. We explored some regions of the parameter space and found rich dynamic behavior, especially when both are periodic. One might argue that periodic opinions and toroidal lattices are not realistic and even artificial. But the opposite might be true: linear opinions and rectangular lattices are rather special cases. The surface of the Earth is finite and has no boundary. Opinions, discrete or continuous, are not always linear either. In some cases, opinions can be better represented by more general structures other than a line. In short, our results show that if we generalize the spaces for opinions and lattices, the dynamic behavior of those systems can be richer.

In reality, a consensus is not reached easily. Consider political opinions as an example. Even though political parties have been formed in advanced societies, people in one party usually have various political views. Another example is the existence of dialects, if we regard languages as opinions. These phenomena can be explained by inherent heterogeneity of agents, or bounded confidence. Our model, however, adds another explanation: metastability through local interactions. The existence of metastable states in our model indicates that locally converged, but not globally converged, states can be sustained for a long time in certain situations. For example, if we are surrounded by like-minded people, we seldom change our views and even believe that everybody is similar to us, which is not true in general.

In general, steady states or equilibrium states in models that are closed are hard to realize in real systems like societies because societies are fundamentally open and extremely noisy. However, the behavior of transient states and emergence of different types of steady state can shed some light on how real systems behave. In this model, metastable states, which have the full spectrum of opinions, are only observed in cases of toroidal lattices with periodic opinions. But even for cases with rectangular lattices, as the size of the lattice gets bigger, the overall behavior of locally converged transient states seen in Fig. 2 will be similar as long as the opinions are periodic. Then we can interpret our real-life states containing a wide spectrum of opinions as transient states that are moving slowly toward steady states.

The model considered here can be expanded or modified. We can assume $g$ and $d$ are not constant throughout the whole population (so-called heterogeneous agents [12,19,25]). The definition of neighbors can be modified by using a bigger range, so that the network structure becomes more realistic.
We can also consider an additional coevolving network like an acquaintance network on top of our model, and it will be investigated in a future presentation.

Models for social dynamics like this are not devised for predicting the future of specific systems, but can help us understand dynamical properties. In addition, they can be adapted to a wide variety of different systems with similar features.

\section{ACKNOWLEDGMENTS}

The author thanks Chang-Yong Lee for his help with analysis of metastable states. The author also thanks L. E. Reichl for her thoughtful comments concerning this manuscript. This work was supported by Kongju National University.

\section{APPENDIX: SOLVING A 1D CASE}

We make the lattice one dimensional by setting $Y=1$, and we also assume $g=1$ and the maximal $d(d=1$ for nonperiodic opinions and $d=0.49$ for periodic opinions). If the density of agents, the average number of agents at each location, is high enough, we can simplify the system by making the opinion a function of the location, ignoring the states of individual agents. That is due to the fact that every agent in the same location will have the same opinion instantly. Then the overall $N$-agent dynamics is reduced to the dynamics of $\phi(x)$, an $X$-dimensional vector.

Since an agent has three choices to move (left, right, and staying) with the probability $1 / 3$ for each move (see Fig. 9), the opinion at $x, \phi(x)$, at the next time step will become $[\phi(x-1)+\phi(x)+\phi(x+1)] / 3$. This is a discrete-time linear dynamical system, which can be characterized by a tridiagonal matrix with all nonzero elements $1 / 3$. Usually we are interested in finding states where $\phi(x)$ does not change with time, and they are eigenstates of this matrix with the eigenvalue 1 . If we look at the dynamics locally, when $\phi(x-1)=\phi(x)-\delta$ and $\phi(x+1)=\phi(x)+\delta, \phi(x)$ will not change at the next time step, where $\delta$ is a small real number at $x$.

Since both $x$ and $\phi$ are bounded, we cannot find eigenstates easily, except in one trivial case when $\phi(x)$ is constant with respect to $x$ ( $\delta=0$ for all $x)$. If a system arrives at these states, a consensus has been reached. In most cases, these are the only eigenstates, but when both the lattice and the opinion are periodic, periodic eigenstates can exist as long as the boundary conditions are met, where $\delta$ is a nonzero constant for all $x$. However, $\delta$ needs to have certain discrete values, and we can name these periodic eigenstates with period numbers. Period $n_{\phi} / n_{l}\left(n_{\phi}, n_{l}=1,2,3, \ldots\right)$ means that opinions have $n_{\phi}$ cycles of changes while there are $n_{l}$ cycles of changes along the $x$ direction; hence only when $\delta=\left(n_{\phi} / n_{l}\right) X$ will they become eigenstates.

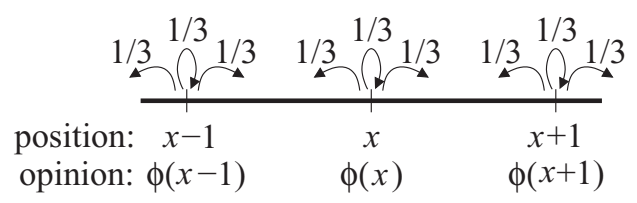

FIG. 9. Schematic diagram for a $1 \mathrm{D}$ case $(Y=1)$ with $g=1$ and the maximal $d$. 
[1] S. Galam, Y. Gefen, and Y. Shapir, J. Math. Sociol. 9, 1 (1982).

[2] C. Castellano, S. Fortunato, and V. Loreto, Rev. Mod. Phys. 81, 591 (2009).

[3] T. C. Schelling, Micromotives and Macrobehavior (W. W. Norton and Company, New York, 1978).

[4] T. M. Liggett, Stochastic Interacting Systems: Contact, Voter and Exclusion Processes (Springer-Verlag, Berlin, 1999).

[5] S. Fortunato and C. Castellano, Phys. Rev. Lett. 99, 138701 (2007).

[6] M. de Oliveira, J. Stat. Phys. 66, 273 (1992).

[7] S. Galam, J. Math. Psychol. 30, 426 (1986); Physica A 336, 56 (2004); Phys. Rev. E 71, 046123 (2005); Europhys. Lett. 70, 705 (2005).

[8] S. Galam, B. Chopard, A. Masselot, and M. Droz, Eur. Phys. J. B 4, 529 (1998).

[9] K. Sznajd-Weron and J. Sznajd, Int. J. Mod. Phys. C 11, 1157 (2000).

[10] A. Ianni and V. Corradi, Rev. Econ. Design 7, 257 (2002).

[11] F. Vazquez, P. L. Krapivsky, and S. Redner, J. Phys. A 36, L61 (2003).

[12] E. Ben-Naim, P. L. Krapivsky, and S. Redner, Physica D 183, 190 (2003).

[13] S. Gil and D. H. Zanette, Phys. Lett. A 356, 89 (2006).

[14] F. Fu and L. Wang, Phys. Rev. E 78, 016104 (2008).

[15] S. Mandrà, S. Fortunato, and C. Castellano, Phys. Rev. E 80, 056105 (2009).

[16] I. J. Benczik, S. Z. Benczik, B. Schmittmann, and R. K. P. Zia, Phys. Rev. E 79, 046104 (2009).
[17] S. Chatterjee and E. Seneta, J. Appl. Prob. 14, 89 (1977).

[18] R. Hegselmann and U. Krause, J. Artif. Soc. Soc. Simul. 5(3), 2 (2002).

[19] G. Weisbuch, G. Deffuant, F. Amblard, and J.-P. Nadal, Complexity 7, 55 (2002)

[20] G. Weisbuch, G. Deffuant, and F. Amblard, Physica A 353, 555 (2005).

[21] S. Fortunato, Physica A 348, 683 (2005).

[22] P. Holme and M. E. J. Newman, Phys. Rev. E 74, 056108 (2006).

[23] J. Lorenz, Int. J. Mod. Phys. C 18, 1819 (2007).

[24] B. Kozma and A. Barrat, J. Phys. A 41, 224020 (2008).

[25] G. Iñiguez, J. Kertész, K. K. Kaski, and R. A. Barrio, Phys. Rev. E 80, 066119 (2009).

[26] R. Axelrod, J. Conflict Resolut. 41, 203 (1997).

[27] M. Laguna, G. Abramson, and D. H. Zanette, Physica A 329, 459 (2003).

[28] F. Vazquez and S. Redner, Europhys. Lett. 78, 18002 (2007).

[29] G. Deffuant, D. Neau, F. Amblard, and G. Weisbuch, Adv. Complex Syst. 3, 87 (2000).

[30] T. Gross and B. Blasius, J. R. Soc. Interface 5, 259 (2008).

[31] J. Stehlé, A. Barrat, and G. Bianconi, Phys. Rev. E 81, 035101 (2010).

[32] W. Feller, An Introduction to Probability Theory and its Applications, 3rd ed. (Wiley, New York, 1965), Vol. I.

[33] L. Festinger, H. W. Riecken, and S. Schachter, When Prophecy Fails (University of Minnesota Press, Minneapolis, 1956).

[34] D. Gal and D. D. Rucker, Psychol. Sci. 21, 1701 (2010).

[35] F. Amblard and G. Deffuant, Physica A 343, 725 (2004). 